\title{
Hipertensión pulmonar y el paciente quirúrgico: Estrategias de manejo actual
}

\author{
MAGDALENA FERMANDOIS C., RODRIGO LÓPEZ B., \\ PAULA LEÓN S., GUILLERMO LEMA F.
}

\author{
Departamento Anestesiología. \\ Facultad de Medicina, \\ Pontificia Universidad Católica \\ de Chile. Santiago de Chile. \\ Recibido el 24 de abril de \\ 2009, aceptado el 6 de \\ octubre de 2009 \\ Correspondencia a: \\ Magdalena Fermandois C \\ Código postal 7770407 \\ Fono: 3543415 \\ E-mail: fermandois@vtr.net
}

\section{Current management strategies for pulmonary hypertension in surgical patients}

Pulmonary hypertension is a clinical condition with a low prevalence, but carries high morbidity and mortality. Important advances in the understanding of this disease and in its therapy have occurred. A particular scenario is pulmonary hypertension in patients undergoing non-cardiac as well as cardiac surgery. The aim of this review is to present information that may allow diagnostic and therapeutic approaches of this clinical condition in patients undergoing surgery.

(Rev Med Chile 2010; 138: 496-503).

Key words: Hypertension, pulmonary; Therapeutics; Thoracic surgery.
L a hipertensión pulmonar (HTP) aguda y crónica es una patología de baja prevalencia pero de alto impacto en la morbilidad y mortalidad. En las últimas dos décadas se han logrado importantes avances en la comprensión de su fisiopatología y en el manejo clínico. En la actualidad, la expectativa de vida de los pacientes con HTP idiopática ha mejorado, haciendo probable que algunos de ellos se presenten para cirugía general.

Existe consenso sobre el manejo de los pacientes con HTP idiopática, sin embargo, el tema no es tan claro para aquellos con HTP de causa conocida y menos aun en el contexto perioperatorio. En este escenario la mayor parte de las sugerencias terapéuticas han sido extrapoladas de la experiencia en pacientes con HTP idiopática ${ }^{1-5}$.

La presente revisión se enfoca en el paciente adulto con HTP en el perioperatorio, su fisiopatología y estrategias de terapias sugeridas.

La definición de HTP incluye: presión de arteria pulmonar media (PAPm) mayor a 25 $\mathrm{mmHg}$ en reposo o a $30 \mathrm{mmHg}$ en ejercicio, con una resistencia vascular pulmonar (RVP) elevada,
$>3$ unidades Wood ${ }^{1}$. El diagnóstico requiere cateterismo cardíaco, ya que confirma el diagnóstico, aporta información pronóstica, y permite evaluar la reactividad vascular pulmonar.

\section{Clínica}

Generalmente son mujeres jóvenes, con deterioro de la capacidad funcional por disnea, palpitaciones, angor (isquemia de ventrículo derecho) y síncope (marcador clínico de mal pronóstico) ${ }^{6,7}$.

La herramienta más útil como método de pesquisa y evaluación no invasiva de HTP es la ecocardiografía. El examen diagnóstico definitivo es el cateterismo cardíaco derecho, el que mide presión de capilar pulmonar (PCP), presión de arteria pulmonar (PAP), presión de ventrículo derecho (VD), presión de aurícula derecha (AD) y oximetría de arteria pulmonar. Con los datos obtenidos, se pueden calcular las variables derivadas, incluyendo la RVP; permite evaluar también la respuesta de la vasculatura pulmonar a la infusión de vasodilatadores ${ }^{4,7-9}$. 
El test de la caminata de los 6 minutos es una prueba de esfuerzo submáximo, útil en la evaluación inicial del paciente con HTP y en su seguimiento. Sus valores basales se correlacionan con la capacidad funcional, parámetros hemodinámicos y sobrevida. Permite además evaluar la respuesta frente a tratamiento ${ }^{4,6-8}$.

\section{Fisiopatología}

El territorio vascular pulmonar se caracteriza por ser de alto flujo y baja resistencia, debido a que sus arterias y arteriolas son de diámetro mayor y de paredes más delgadas que sus correspondientes sistémicas. La reactividad de la vasculatura pulmonar está determinada principalmente por el endotelio, que es el efector común a diferentes estímulos capaces de gatillar HTP. Se ha descrito que el shear stress, hipoxia, síndrome de distress agudo pulmonar y sepsis, son capaces de activar el endotelio en sujetos genéticamente susceptibles, lo que genera vasoconstricción pulmonar y estimulación de la proliferación celular ${ }^{1,5,10}$ (Tabla 1).

Desde el punto de vista hemodinámico, frente a un aumento agudo de la PAP, el VD responde generando presiones hasta de 40 a $60 \mathrm{mmHg}$ y aumenta el volumen de fin de diástole. Cuando los mecanismos de compensación aguda son superados, cae el gasto cardíaco (GC) y se produce insuficiencia cardíaca. Frente a una sobrecarga crónica de presión, la compensación es a través de hipertrofia, la que puede generar alteración de la contractilidad y mayor susceptibilidad de isquemia. En un intento por mejorar el GC, se aumenta la precarga, lo que genera dilatación de anillo valvular tricuspídeo e insuficiencia cardíaca ${ }^{1,4,11}$.

Tabla 1. Estímulos para el endotelio que gatillan HTP

\begin{tabular}{|l}
\hline - Hipoxia \\
\hline - Acidosis \\
- Radicales libres \\
- Mediadores inflamatorios \\
- Aumento del flujo sanguíneo pulmonar \\
- Fibrina ( liberada en tromboembolismo) \\
- Microembolías
\end{tabular}

\section{Factores agravantes de HTP}

Dióxido de carbono/acidosis. Un aumento del $\mathrm{CO}_{2}$ indirectamente aumenta la resistencia vascular pulmonar, debido al aumento de la concentración de hidrogeniones. Esto hace particularmente frágiles a estos pacientes frente a la hipoventilación alveolar ${ }^{12}$.

Tensión de oxígeno. Existe una relación no lineal entre la tensión alveolar de oxígeno y la resistencia vascular pulmonar, la cual aumenta cuando la $\mathrm{PaO}_{2}$ disminuye bajo $60 \mathrm{mmHg}$. Dicho aumento es potenciado por la acidosis. Este fenómeno, conocido como vasoconstricción pulmonar hipóxica $(\mathrm{VPH})$, regula de manera aguda la relación ventilación/perfusión, desviando el flujo sanguíneo de las áreas que no están siendo ventiladas ${ }^{13}$.

Volumen pulmonar. Los vasos pulmonares pueden ser comprimidos tanto por aire en los alvéolos (sobredistención) como por el parénquima (volúmenes pulmonares bajos). En ambos casos aumenta la RVP (Figura 1).

Óxido nítrico (NO). Es un mensajero con múltiples acciones, de las cuales la más importante a nivel pulmonar es mantener el tono vascular basal. Se sintetiza a partir de la L-arginina, a través de la óxido-nítrico sintetasa (NOs). Aumenta la concentración intracelular de GMPc, relajando al músculo liso (Figura 2). La NOs es regulada por estímulos como el shear stress y el flujo pulmonar. Su función es regular la vasoconstricción pulmonar hipóxica, inhibir la proliferación del músculo liso, la agregación plaquetaria y la producción de endotelina $1^{10,14}$.

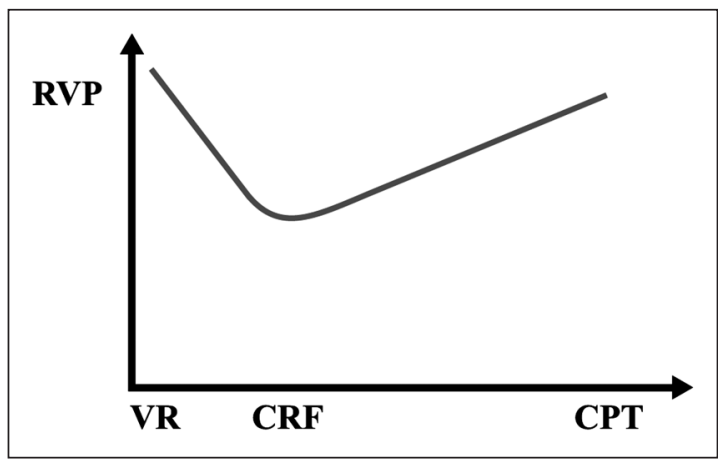

Figura 1. Relación entre RVPy volúmenes pulmonares. Leyenda: VR: volumen residual, CRF: capacidad residual funcional, CPT: capacidad pulmonar total. 


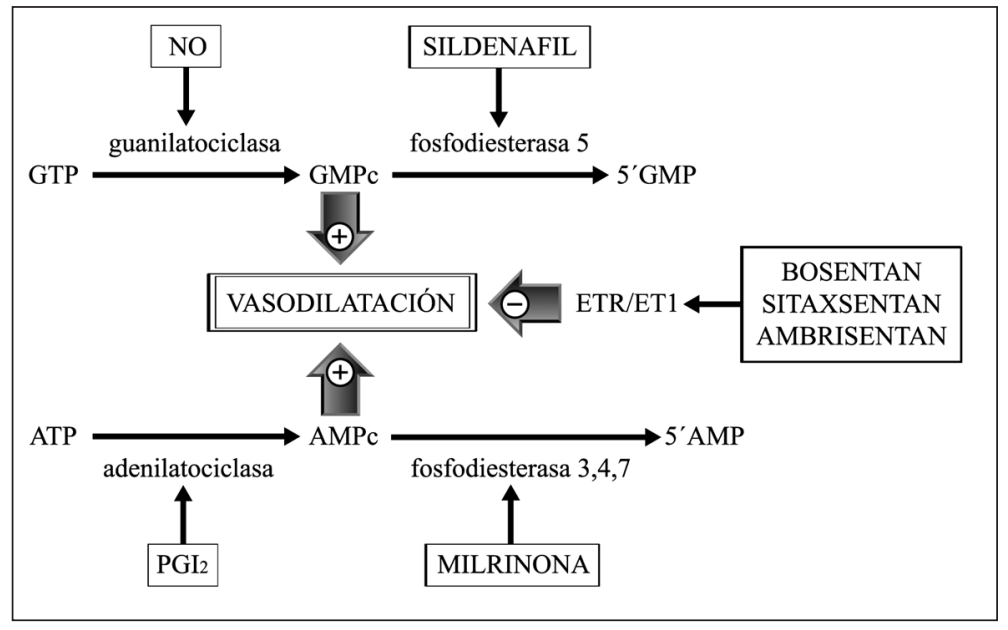

Figura 2. Vías implicadas en la HTP y los fármacos que actúan en ella.
Prostaciclina (PGI $)$. Metabolito del ácido araquidónico, producido por células endoteliales y musculares lisas, es vasodilatador y antiagregante plaquetario, sintetizado en respuesta a la vasoconstricción. Pacientes con HTP de diversas causas tienen síntesis de $\mathrm{PGI}_{2}$ disminuida comparado con controles sanos ${ }^{4}$.

Endotelina $1\left(E T_{1}\right)$. Potente vasoconstrictor sintetizado por el endotelio, clave en la patogénesis y progresión de la HTP. Su expresión está aumentada en estos pacientes y sus niveles se correlacionan con la alteración hemodinámica pulmonar ${ }^{15}$. Actúa uniéndose a receptores específicos de membrana: ET-A y ET-B.

\section{Agentes terapéuticos potenciales}

1. Óxido nítrico: Su administración vía inhalatoria permite que se relaje la vasculatura pulmonar sin hipotensión sistémica y alcanzar los alvéolos abiertos, disminuyendo el shunt. Requiere de un sistema especial de administración, con personal capacitado, haciéndolo difícil de implementar en pabellón. Está aprobado su uso en prematuros con HTP e insuficiencia respiratoria; su utilidad en adultos es menos clara, teniendo un rol en el test de vasorreactividad y en la HTP perioperatoria ${ }^{1,16}$.

2. Sildenafil: Inhibidor selectivo de la fosfodiesterasa 5, responsable de la hidrólisis intracelular del GMPc. Actúa potenciando la relajación mediada por NO al disminuir la degradación del GMPc. Lewis et al evaluaron el impacto en la capa- cidad de ejercicio y la calidad de vida de sildenafil en pacientes con HTP secundaria a insuficiencia cardíaca sistólica, y luego de 12 semanas disminuyó significativamente la RVP en reposo y ejercicio, junto a un aumento del volumen expulsivo, lo que se vio reflejado en una mejoría clínica ${ }^{17}$. En pacientes con HTP secundaria a disfunción crónica del ventrículo izquierdo, disminuyó la RVP en ejercicio y mejoró el consumo máximo de oxígeno luego de administración única ${ }^{18}$.

\section{Antagonistas de endotelina}

3a. Selectivos del receptor ET-A:

Ambrisentan: Se evaluó su repercusión clínica en pacientes con HTP idiopática, en quienes mejoró la clínica, con buen perfil hepático ${ }^{19}$.

Sitaxsentan: Estudio STRIDE-2 mostró mejoría de la capacidad de ejercicio, con baja incidencia de alteraciones hepáticas ${ }^{20}$.

\section{3b. No selectivos de ET-A:}

Bosentan: Antagonista competitivo de los receptores ET-A y B, con una afinidad de 20:1 respectivamente. Disminuye la remodelación de la vasculatura pulmonar. Su efectividad en pacientes con HTP idiopática ha sido evaluada en estudios contra placebo ${ }^{21}$, demostrándose mejoría de la capacidad funcional, menor disnea, mayor índice cardíaco (IC) y disminución de la RVP. La mejoría clínica se ha correlacionado con un aumento de la fracción de eyección del VD. La evidencia sugiere un beneficio en la sobrevida, pero faltan estudios prospectivos y aleatorios que lo demuestren. 


\section{Prostanoides (análogos de prostaciclina):}

4a. Epoprostenol: Primera terapia que modificó el pronóstico de la HTP idiopática ${ }^{9,10}$. Su administración es a través de vía venosa central. Su efecto sería mediado por vasodilatación, inhibición de proliferación del músculo liso vascular, antiagregación plaquetaria, disminución de alteración endotelial, y mejoría de la hemostasis. Se ha usado vía inhalatoria en pacientes quirúrgicos que han presentado insuficiencia ventricular derecha, con resultados poco claros ${ }^{22}$.

4b. Iloprost: Análogo sintético de la prostaciclina, efectivo en el manejo de pacientes con hipertensión pulmonar ${ }^{23}$. En pacientes con HTP secundaria a valvulopatía mitral que persistió luego de la reparación valvular, se observó disminución significativa de la PAPm y de la RVP, aumento del IC, sin modificación de la presión arterial media ni de la resistencia vascular sistémica (RVS ${ }^{24}$. En el período perioperatorio es una droga atractiva, ya que es efectivo como la PGI intravenosa pero con mínimos efectos sistémicos ${ }^{\frac{2}{3,24,25}}$, de fácil administración, no requiere accesos venosos centrales, se puede nebulizar a través de la línea inspiratoria del circuito ventilatorio de los pacientes intubados y se puede continuar nebulizando después de la extubación. El año 2000, el Grupo Alemán para Estudio de la HTP comparó durante cateterismo cardíaco de pacientes con HTP idiopática, la respuesta hemodinámica frente a $\mathrm{NO}$ e iloprost: la PAM disminuyó con iloprost pero la PAS no bajo de los $90 \mathrm{mmHg}$, el GC, IC, volumen expulsivo, RVP mejoraron con NO respecto de los niveles basales, pero mejoraron aun más con iloprost; la $\mathrm{paO}_{2}$ mejoró con iloprost y no con $\mathrm{NO}^{25}$.

5. Magnesio: Actúa como antagonista del calcio, produciendo relajación muscular. Estimula síntesis de NO, aumenta el AMPc y la liberación de $\mathrm{PGI}_{2}{ }^{1}$.

6. Inótropos y vasopresores: Las drogas vasoactivas que tiene el mejor perfil para el manejo del paciente con HTP son la milrinona y la norepinefrina.

6a. Milrinona: Actúa aumentando el AMPc, lo que aumenta la contractilidad y disminuye la RVP. De administración intravenosa. Indicada en pacientes con aumento de la RVP asociada a disminución de la contractilidad ventricular. Se ha reportado su uso a través de nebulización, para obtener efecto vasodilatador vascular pulmonar, evitando la hipotensión sistémica ${ }^{26}$.

6b. Norepinefrina: La norepinefrina se comporta como vasoconstrictor en el territorio sistémico por sobre el pulmonar, haciéndola útil en el tratamiento de la falla ventricular derecha secundaria a HTP aguda ${ }^{1}$.

7. Antagonistas del calcio: Los pacientes candidatos a recibir este tratamiento son sólo aquellos que han sido considerados como respondedores al test vasodilatador.

8. Adenosina: Actúa directamente sobre las células musculares lisas e induce relajación al aumentar la liberación de NO. Su vida media es de 9 segundos, por lo que su acción alcanza a ocurrir sólo en el territorio pulmonar. Utilizada principalmente en el test de vaso reactividad.

\section{Escenarios quirúrgicos posibles}

\section{A. Paciente con diagnóstico de HTP que se presenta a cirugía no cardíaca}

Ramakrishna evaluó la morbilidad y mortalidad a 30 días de 145 pacientes con diagnóstico preoperatorio de HTP (no secundaria a enfermedad de corazón izquierdo) que se sometieron a cirugía no cardíaca bajo anestesia general, $42 \%$ tuvo al menos 2 eventos mórbidos y la mortalidad fue de 7\%. Las principales causas de morbi/mortalidad fueron insuficiencia respiratoria y cardíaca ${ }^{27}$. Se han identificado algunas variables predictoras independientes de eventos adversos ${ }^{3,28}$ (Tabla 2).

Se han evaluado diferentes técnicas anestésicas: sedación, anestesia general inhalatoria o intrave-

Tabla 2. Predictores de resultados adversos

HTP suprasistémica

Historia de embolia pulmonar

Capacidad funcional mayor o igual a II

Duración de anestesia mayor a tres horas

Cirugía de riesgo intermedio o alto (abdominal mayor, traumatológica, torácica, vascular, trasplante) 
nosa, sin diferencias en la morbi/mortalidad entre ellas ${ }^{28}$. La sedación debe ser titulada para cada paciente; su beneficio sería disminuir la actividad simpática secundaria al estrés perioperatorio, pero conlleva el riesgo de desaturación y retención de $\mathrm{CO}_{2}$. Con respecto a la anestesia neuroaxial, hay datos aportados desde la anestesia obstétrica. A pesar de que la anestesia peridural posee el riesgo teórico de disminuir el GC por caída del retorno venoso, no se ha asociado a mayor mortalidad de este grupo de pacientes ${ }^{29}$. La anestesia espinal, por sus cambios hemodinámicos bruscos, no es recomendable, siendo una alternativa atractiva la anestesia mixta (general más epidural).

En cirugía electiva, se debe decidir en conjunto con todos los especialistas involucrados en el manejo del paciente los exámenes preoperatorios requeridos, el tratamiento óptimo y el pronóstico, considerando el estado preoperatorio y la cirugía a la que va a ser sometido.

Existen diferentes regímenes terapéuticos dependiendo del estado en que se encuentre el paciente, de la reactividad que haya mostrado frente a vasodilatadores, de la capacidad funcional y de la experiencia del equipo tratante. Es importante mantener hasta la cirugía el esquema que le ha dado mejor estabilidad clínica. Se debe considerar la evolución del paciente, para poder planificar la terapia frente a una crisis perioperatoria ${ }^{3}$.

Es recomendable que la monitorización sea invasiva, con presión arterial directa, catéter de arteria pulmonar (CAP), ecocardiografía transesofágica $(\mathrm{ETE})^{3,30}$, o ambas. Esta última es una herramienta particularmente útil en este contexto, ya que es fácil de implementar, de baja morbilidad y se evitan las arritmias que genera el paso de un CAP, que pueden ser mal toleradas. Considerar que el CAP permite monitorizar de manera continua en el postoperatorio, lo que no es posible con el ETE.

Como metas generales se debe mantener controlada la PAP, la precarga ventricular derecha, y evitar la isquemia del VD.

En el extremo del espectro clínico del paciente con HTP, se encuentra el síndrome de Eisenmenger. Este corresponde a pacientes con cardiopatía congénita que inicialmente presentaron cortocircuito de izquierda a derecha, y que luego de remodelación vascular pulmonar se produjo un aumento de la RVP, invirtiendo el cortocircuito (de derecha a izquierda). Si bien son pacientes con

\section{Tabla 3. Situaciones asociadas a riesgo de hipertensión pulmonar}

Tromboembolismo pulmonar (TEP)

Embolia de $\mathrm{CO}_{2}$ (cirugía laparoscópica)

Embolia aérea (posición quirúrgica sentada, cesárea, artroplastía total de cadera, instalación de catéter venoso central, infusiones intravenosas)

Cementación óseo en cirugía traumatológica

Síndrome de reperfusión

alta morbilidad y mortalidad perioperatoria, las estrategias de manejo son las mismas que hemos delineado para el paciente con HTP; cabe destacar que en esta situación es de especial importancia evitar la caída de la RVS, siendo prudente iniciar vasopresores desde el momento de la inducción anestésica.

\section{B. Paciente sin HTP conocida, que se presenta a cirugía general}

Evento clínico de baja incidencia, pero de gran repercusión, reportándose tasas a 30 días de $42 \%$ de morbilidad y $9,7 \%$ de mortalidad ${ }^{3}$. Por lo tanto, es importante tener un alto grado de sospecha frente a situaciones de shock cardiovascular inesperado.

Circunstancias asociadas a riesgo de hipertensión pulmonar se mencionan en Tabla $3^{1,31}$.

Los pacientes que se encuentren en riesgo de trombosis venosa profunda (TVP) como pacientes con neoplasia intercurrente, ancianos, postrados, o con antecedentes de TVP-TEP, debiesen ser evaluados con un alto grado de sospecha.

Los pacientes obesos sometidos a cirugía bariátrica presentan factores de riesgo de enfermedad cardiovascular y de enfermedad tromboembólica. El TEP, con una incidencia menor a $0,8 \%$, da cuenta de la mayor parte de la mortalidad de esta cirugía. Se ha asociado el índice de masa corporal (IMC) a una mayor PAP media y a una $\mathrm{PaO}_{2}$ menor $^{32}$. Un IMC mayor a 35 es un signo clínico de riesgo para síndrome de apnea obstructiva del sueño ${ }^{33}$. Este síndrome, la hipoxemia y la hipercapnia son determinantes independientes para desarrollar HTP diurna. 


\section{Paciente sin HTP conocida que se presenta a cirugía cardíaca}

Los pacientes que se presentan a cirugía cardíaca (para revascularización miocárdica o reparación valvular), tienen una aproximación perioperatoria diferente, ya que la patología basal les da un riesgo conocido de presentar esta complicación ${ }^{34}$. En la evaluación preoperatoria, la ecocardiografía evidenciará el grado de afección valvular, presencia y gravedad de disfunción diastólica y sistólica, y repercusión en el territorio pulmonar.

\section{Consideraciones generales del manejo}

En la preparación para cirugía electiva, se debe mantener el tratamiento durante el perioperatorio. Es conveniente verificar con qué terapias de rescate cuenta la institución y cuales son las posibilidades de implementarlas en el pabellón.

El estrés perioperatorio, a través de la liberación de catecolaminas, genera incrementos mal tolerados de la presión arterial pulmonar. Como objetivos hemodinámicos generales, evitar: aumentos de la RVP, disminución del retorno venoso, disminución de la RVS y depresores miocárdicos ${ }^{29}$. Si fuese necesario un soporte vasopresor, recordar que la norepinefrina tiene el perfil más adecuado para el manejo de estos pacientes.

Con respecto a la elección de anestésicos intravenosos, el propofol y el tiopental parecen ser seguros en pacientes con HTP; la ketamina debiera evitarse ya que podría aumentar la $\mathrm{RVP}^{1}$. Bajo anestesia general, los halogenados podrían disminuir la VPH pero sin repercusión clínica importante ${ }^{13}$. Más importante que la droga utilizada, es que la concentración tenga mínima repercusión hemodinámica.

Pacientes con HTP venosa empeoran su condición con vasodilatadores pulmonares ya que estos producen edema pulmonar. Se debe optimizar el manejo de la insuficiencia cardíaca izquierda.

Se debe planificar el postoperatorio en unidad de cuidados intensivos ( período de máxima mortalidad $)^{3,27,29}$. Si la HTP se considera en el preoperatorio como severa, la cirugía es de gran magnitud o el paciente tiene un importante componente reactivo en el intraoperatorio, se debe considerar sedación profunda, ventilación mecánica e incluso parálisis neuromuscular durante el postoperatorio. La sedación y analgesia profunda se pueden lograr con infusiones de opioides y benzodiacepinas; esto permite disminuir la respuesta simpática que puede gatillar eventos de HTP reactiva, especialmente en las primeras horas del postoperatorio. La ventilación mecánica debe asegurar una oxigenación adecuada (PEEP 5 a $10 \mathrm{cmH} O$ y fracción inspirada de $\mathrm{O}_{2}$ alta) y mantener un $\mathrm{CO}_{2}$ en rango normal bajo.

\section{Sugerencias terapéuticas}

Como aproximación básica frente a todo paciente con una crisis de hipertensión pulmonar, se sugiere:

- Si la HTP se diagnostica en el preoperatorio y la cirugía es impostergable, iniciar tratamiento con sildenafil.

- Dar $\mathrm{FiO}_{2}$ 100\%.

- Mantener el $\mathrm{CO}_{2}$ teleespiratorio en rango normal bajo (30-35 $\mathrm{mmHg}$ ).

- Mantener el pH normal, si no es suficiente con la ventilación, agregar bicarbonato.

- Evitar tanto el colapso pulmonar como la sobredistención.

- Mantener una adecuada profundidad anestésica y analgésica.

- Mantener temperatura normal.

Entre los diferentes fármacos, considerar que diferentes mecanismos de acción implican efectos al menos aditivos, si no sinérgicos.

- Asegurar normovolemia y adecuada presión de perfusión para el miocardio (preferir noradrenalina).

- Si la presión arterial sistémica se logra mantener con noradrenalina, se puede agregar milrinona intravenosa.

- Si la HTP no disminuye, y aún compromete la hemodinámica, se pueden iniciar nebulizaciones con iloprost.

\section{Referencias}

1. Fischer L, Van Aken H, Burkle H. Management of pulmonary hypertension. Anesth Analg 2003; 96: 1603-16.

2. Rich S, Rabinovitch M. Diagnosis and treatment of secondary (non-category 1) pulmonary hypertension. Circulation 2008; 118: 2190-9.

3. Fox C, Kalarickal P, Yarborough M, Jin J. Perioperative 
management including new pharmacological vistas for patients with pulmonary hypertension for non cardiac surgery. Curr Opin Anesthesiol 2008; 21: 467-72.

4. Blaise G, Langleben D, Hubert B. Pulmonary arterial hypertension pathophysiology and anesthetic approach. Anesthesiology 2003; 99: 1415-32.

5. Simonneau G, Galié N, Rubin L, Langleben D, Seeger W, Domenighetti G, et al. Clinical classification of pulmonary hypertension. J Am Coll Cardiol 2004; 43: 5S-12S.

6. Faber H, Loscalzo J. Pulmonary arterial hypertension. N Engl J Med 2004; 351: 1655-65.

7. Zagolín M, Wainstein E, Uriarte P. Actualización en el diagnóstico y terapéutica en hipertensión pulmonar arterial. Rev Med Chile 2006; 134: 902-9.

8. Carrión JL, Vicente R, Rodríguez G. Hipertensión arterial pulmonar: fisiopatología, diagnóstico, tratamiento y consideraciones anestésicas. Rev Esp Anestesiol Reanim 2007; 54: 93-108.

9. Coghlan G, Corris P, Gaine S, Gatzoulis M, Gibbs S, Haworth S, et al. Consensus statement on the management of pulmonary hypertension in clinical practice in the UK and Ireland. Thorax 2008; 63 (suppl II): iil-ii41

10. Budhiraja R, TuderR, Hassoun P. Endothelial dysfunction in pulmonary hypertension. Circulation 2004; 109: 159-65.

11. Hemnes A, Champion H. Right heart function and hemodynamics in pulmonary hypertension. Int J Clin Pract 2008; 62: 11-9.

12. Chang AC, Zucker HA, Hickey PR, Wessel D. Pulmonary vascular resistance in infants after cardiac surgery: Role of carbon dioxide and hydrogen ion. Crit Care Med 1995; 23: 568-74.

13. Grichnik K, Clark J. Pathophysiology and management of one-lung ventilation. Thorac Surg Clin 2005; 15: 85103.

14. Culotta E, Koshland D. No news is good news. Science 1992; 258: 1862-5.

15. Giaid A, Yanagisawa M, Langleben D, Michel R, Levy R, Shennib $\mathrm{H}$, et al. Expression of endothelin-1 in the lungs of patients with pulmonary hypertension. N Engl J Med 1993; 328: 1732-9.

16. Haddad E, Lowson S, Johns R, Rich G. Use of inhaled nitric oxide perioperatively and in intensive care patients. Anesthesiology 2000; 92: 1821-5.

17. Lewis G, Shah R, Shahzad K, Camuso J, Pappagianopoulos P, Hung J, et al. Sildenafil improves excercise capacity and quality of life in patients with systolic heart failure and secondary pulmonary arterial hypertension. Circulation 2007; 116: 1555-62.

18. Lewis G, Lachmann J, Camuso J, Lepore J, Shin J, Martinovic $\mathrm{M}$, et al. Sildenafil improves exercise hemodyna- mics and oxygen uptake in patients with systolic heart failure. Circulation 2007; 115: 59-66.

19. Galie N, Olschewski H, Oudiz R, Torres F, Frost A. Ambrisentan for the treatment of pulmonary arterial hypertension. Circulation 2008; 117: 3010-9.

20. Barst RJ, Langleben D, Badesch D, Frost A, Lawrence EC, Shapiro S, et al. Treatment of pulmonary arterial hypertension with the selective endothelin-A receptor antagonist sitaxsentan. J Am Coll Cardiol 2006; 47: 2049-56.

21. Rubin L, Badesch D, Barst R, Galiè N, Black C, Keogh A, et al. Bosentan therapy for pulmonary arterial Hypertension. N Engl J Med 2002; 346: 896-903.

22. Schroeder R, Wood G, Plotkin J, Kuo P. Intraoperative Use of inhaled $\mathrm{PGI}_{2}$ for acute pulmonary hypertension and right ventricular failure. Anesth Analg 2000; 91: 291-5.

23. Olschewski H, Simonneau G, Galié N, Higenbottam T, Naeije R, Rubin L, et al. Inhaled iloprost for severe pulmonary hypertension. N Engl J Med 2002; 347: 322-9.

24. Langer F, Wilhem W, Tscholl D, Schramm R, Lausberg $\mathrm{H}$, Wendler $\mathrm{O}$, et al. Intraoperative inhalation of the long acting prostacyclin analog iloprost for pulmonary Hypertension. J Thorac Cardiovasc Surg 2003; 126: 874-5.

25. Hoeper, M, Olschewski H, Ghofrani H, Wilkens H, Winkler J, Borst $\mathrm{M}$, et al. A comparison of the acute hemodynamic effects of inhaled nitric oxide and aerosolized iloprost in primary pulmonary hypertension. J Am Coll Cardiol 2000; 35; 176-82.

26. Buckley M, Feldman J. Nebulized milrinone use in a pulmonary hypertensive crisis. Pharmacotherapy 2007; 27: 1763-6.

27. Ramakrishna G, Sprung J, Ravi B, Chandrasekaran K, McGoon M. Impact of pulmonary hypertension on the outcomes of noncardiac surgery: Predictors of perioperative morbidity and mortality. J Am Coll Cardiol 2005; 45: 1691-9.

28. Carmosino M, Friesen R, Doran A, Ivy D. Perioperative complications in children with pulmonary hypertension undergoing noncardiac surgery or cardiac catheterization. Anesth Analg 2007; 104: 521-7.

29. Kahn M, Bhatt S, Kryc J. Anesthetics considerations for parturients with primary pulmonary hypertension. Int J Obstet Anesth 1996; 5: 36-42.

30. Rosenkranz S. Pulmonary hypertension: Current diagnosis and treatment. Clin Res Cardiol 2007; 96: 527-41.

31. Mirski M, Lele A, Fitzsimmons L, Toung T. Diagnosis and treatment of vascular air embolism. Anesthesiology 2007; 106: 164-77.

32. Kaw R, Aboussouan L, Auckley D, Bae C, Gugliotti D, Grant $\mathrm{P}$ et al. Challenges in pulmonary risk assessment 
Hipertensión pulmonar y el paciente quirúrgico: Estrategias de manejo actual - M. Fermandois C. et al

and perioperative management In bariatric surgery. Obes Surg 2008; 18: 134-8.

33. Gross J, Bachenberg K, Benumof J, Caplan R, Connis R, Coté $\mathrm{CH}$, et al. Practice guidelines for the perioperative management of patients with obstructive sleep apnea: A report by the American Society of Anesthesiologists
Task Force on perioperative management of patients with obstructive sleep apnea. Anesthesiology 2006; 104: 1081-93.

34. Kaplan J. Kaplan's cardiac anesthesia. Kaplan J, Reich D, Lake C, Konstadt S, editores. Philadelphia, Estados Unidos: Editorial Elsevier 2006; p 645-82. 